\title{
Correlation of HMGB1, PON-1, MCP-1, and Periodontal $P$. gingivalis with Amniotic Fluid Fecal Dye
}

\author{
Zhen-Ai Jin ${ }^{1},{ }^{1}$ Ying Li, ${ }^{1}$ Wei-Bing Chen, ${ }^{2}$ Yu-Ying Wang, ${ }^{3}$ Yi-Kun Zhao, ${ }^{2}$ Xiang-Lan Sun, ${ }^{4}$ \\ Jia-Jun He, ${ }^{4}$ Guo Jie, ${ }^{5}$ and Yu-Mei Sun ${ }^{6}$ \\ ${ }^{1}$ Department of Pediatrics, Affiliated Hospital of Yanbian University, 1327 Bureau Street, Jilin, Yanji, China \\ ${ }^{2}$ Neonatology Department, Rizhao People’s Hospital of Jining Medical University, No. 126, Taian Road, Rizhao, Shandong, China \\ ${ }^{3}$ Jilin Provincial People's Hospital, 1183 Gongnong Street, Chaoyang, Changchun, China \\ ${ }^{4}$ Obstetrical Department, Rizhao People's Hospital of Jining Medical University, No. 126,Taian Road, Rizhao, Shandong, China \\ ${ }^{5}$ Department of Obstetrics, Affiliated Hospital of Yanbian University, 1327 Bureau Street, Yanji, Jilin, China \\ ${ }^{6}$ Department of Neonatology, Dalian Women and Children's Medical Center (Group), No. 1 Dunhuang Road, \\ Dalian Liaoning, China
}

Correspondence should be addressed to Zhen-Ai Jin; 9000001060@ybu.edu.cn

Received 29 November 2021; Revised 23 December 2021; Accepted 28 December 2021; Published 22 February 2022

Academic Editor: Gaurav Goyal

Copyright (c) 2022 Zhen-Ai Jin et al. This is an open access article distributed under the Creative Commons Attribution License, which permits unrestricted use, distribution, and reproduction in any medium, provided the original work is properly cited.

Background. This paper aims to investigate the correlation between high mobility group protein-1 (HMG-b1), antioxidant enzyme-1 (paraoxon-1, PON-1), monocyte chemoattractant protein-1 (monocyte chemoattractant protein-1, MCP-1), P. gingivalis, and MSAF. Materials and Methods. The total sample size comprised of 73 cases in both groups. These patients were further subdivided into 2 groups: the MSAF group and the control group. 38 women were in the MSAF group and 35 women with term amniotic fluid serum were in the control group. The MSAF group was selected as a full-term singleton amniotic fluid fecal infection group. Clinical data were collected, and specimens were collected. Fecal staining of amniotic fluid and full-term amniotic fluid removes the placenta and umbilical cord blood. The expression of HMGB1 in the placenta was observed by immune-histochemical staining of MSAF and control groups. The content of PON-1 in cord blood was determined by ELISA. Results. Correlation between maternal and neonatal clinical data and MSAF was done; MSAF group mean gestational age was $41.38 \pm 1.40$ weeks; control group mean gestational age was $39.20 \pm 1.24$ weeks. This study found no correlation between the birth weight, maternal age, sex, first/transmaternal, hyperthyroidism, hypothyroidism, and anemia between the MSAF and control group with nonsignificant $P$ value $(P>0.05)$. However, the fatal age, gestational diabetes, gestational hypertension, umbilical cord abnormalities, placental abnormalities, and neonatal asphyxia factors were statistically different with a significant $P$ value of $<0.05$ between both groups. HMGB1 and Periodontal $P$. gingivalis are mostly expressed in placental trophoblast, vascular endothelial cells, and amniotic epithelial and interstitial cells. After HE staining of 72 placentas by HE in MSAF and control, 6 had acute chorioamnionitis (5.1 control), 32 had chronic (23.9), 35 had abnormal placentas, and three in MSAF had chorionic columnar metaplasia. In immune-histochemistry experiments, the HMGB1 expression intensity of placental tissue was higher in the MSAF group $(P<0.05)$; however, the level of PON-1 was lower in the MSAF group as compared to the controls $(P<0.05)$. Conclusions. Gestational age and placental abnormalities are clinical high-risk factors for MSAF. HMGB1, PON-1, MCP-1, and Periodontal P. gingivalis may be involved in the development of MSAF, suggesting an oxidative/antioxidant imbalance with inflammation, and may be one of the mechanisms for MSAF development.

\section{Introduction}

In recent years, the birth rate of high-risk pregnant women and critical newborns has been increasing, and the perinatal period of pregnant women has become the focus of attention.
Clinically, the MSAF is defined as follows: when stimulated by a variety of causes, it makes fetal intestinal peristalsis, anal sphincter tension decreases, fetal meconium with intestinal peristalsis into the amniotic fluid, originally bright, and translucent amniotic fluid presents different degrees of 
turbidity, thick, and with the degree of difference, color can appear light green to dark brown change $[1,2]$. The theory of the cause and mechanism of MSAF is not unified. At present, there are theories of fetal maturity and umbilical cord extrusion at birth, as well as the theory of fetal intrauterine distress put forward by many scholars. Meconium-stained amniotic fluid (MSAF) occurs when there is a passage of the fetal colonic contents into the amniotic cavity. The frequency of this condition increases as a function of gestational age. The frequency of MSAF ranges from $5 \%$ to $20 \%$ [3].

The presence of meconium predisposes to meconium aspiration syndrome (MAS) which only occurs in $5 \%$ of all neonates born to mothers with MSAF. MSAF is a risk factor for clinical chorioamnionitis, neonatal hypoxic-ischemic encephalopathy, neonatal sepsis, seizures, and cerebral palsy. Therefore, the presence of MSAF is considered a warning sign by obstetricians, even though most neonates do not have evidence of hypoxia or metabolic academia [4]. MSAF can lead to complications such as intrauterine hypoxia and neonatal asphyxia; due to continuous hypoxia of irreversible damage to brain nerves and other organs, such as meconium inhalation syndrome (meconium aspiration syndrome, MAS), hypoxia and ischemic encephalopathy, etc., can be life-threatening when severe [4]. Wu Meiyan's [5] study pointed out that the amniotic fluid fecal infection is more prone to meconium inhalation syndrome, which further revealed that it is more prone to poor prognosis in newborns. At present, few related studies of MSAF and intrauterine inflammation have been reported. The etiology and pathogenesis of MSAF have not been clarified, and increasing attention has been paid to the search for MSAF markers. Through studies of the placenta and umbilical cord blood-related problems, the correlation between intrauterine inflammation and amniotic fluid fecal infection is of certain research value and significance.

Studies have pointed out that [6] MSAF patients had a chorionic amnionitis (chorioamnionitis, $\mathrm{CA}$ ) probability of more than $50 \%$ associated with intrauterine infection. CA is one of the risk factors of intrauterine infection, and microbes entering the amniotic cavity may cause MSAF, while inflammation of the placenta may cause MSAF. Placental lesions can be classified into acute and chronic placental lesions. Acute lesions are mostly related to infection, and chronic placental lesions could be caused by bacteria, viruses, environmental pollution, and other related factors. In Chen Lifen et al. [7], the length of MSAF was found to be extremely closely related to placental lesions. Some researchers believe that intrauterine inflammation is not related to MSAF, so there is some controversy. In clinical work, intrauterine inflammation can be attributed to placental inflammation, umbilical cord inflammation, and fetal membrane inflammation. Studying its interlocality with MSAF can further investigate the occurrence mechanism of MSAF development.

HMGB1, PON-1, MCP-1, and Periodontal P. gingivalis are inflammatory factors studied associated with MSAF. All these factors have been involved in the occurrence of inflammation, related to oxidative damage mechanism; however, very less studies and reports are conducted in this regard.

HMGB1, one member of the Alertin family, induces the occurrence of an inflammatory response or the repair of stress trauma to initiate host defense. Relevant studies have found that the generation and release of cytokines can change the signaling regulation inside and outside the cell, thus leading to the occurrence of inflammation and the repair after stimulation [8]. Qiu Xiaoyuan [9] believed that increased levels of HMGB1 expression can aggravate the inflammatory response in the placenta. When the body encounters an infection, HMGB1 can be secreted by immune cells. The signaling pathway is initiated by triggering an inflammatory response by binding to the Toll-like receptor 4 (Toll-like receptor 4, TLR4), as well as to the late glycosylation end-product receptor (Receptor for advanced glycation end products, RAGE).

PON-1 is a member of the antioxidant enzyme family with anti-inflammatory effects, associated with antioxidative stress and with the pathogenesis of numerous diseases. The amount of PON-1 at the site of expression is reduced, causing an oxidative stress response, leading to the occurrence of inflammation [10].

MCP-1 is a chemokine with chemotactic monocytes, macrophages, and $\mathrm{T}$ lymphocytes, involved in the inflammatory response, associated with apoptosis, the occurrence and progression of pregnancy diseases, and premature fetal membrane rupture.

Periodontitis in pregnancy should not be confused with pregnancy gingivitis. Pregnancy gingivitis is a common, reversible condition of gingival inflammation associated with high levels of estrogen and blooms of microbial species such as $P$. gingivalis. In periodontitis, the modification of the microbial composition is unrelated to pregnancy status or pregnancy hormones. When good oral hygiene practices are implemented, pregnancy gingivitis resolves within a few months of birth with no permanent changes in CAL. The presence of microbial invasion of the amniotic cavity by $P$. gingivalis could indicate a role for periodontal pathogenic bacteria in pregnant women with a diagnosis of threatened premature labor.

In this study, clinical risk factors of MSAF were analyzed by maternal and neonatal clinical data. The basic aim of the study was to explore the correlation of HMGB1, PON-1, MCP-1, and P. gingivalis with MSAF.

\section{Materials and Methods}

2.1. Collection of Clinical Data Specimens and Data. Clinical case data of amniotic fluid fecal-stained newborn and normal newborns born were collected from the Affiliated Hospital of Yanbian University.

Placenta and cord blood from the term women with an MSAF collection were set as MSAF groups, while women with term amniotic fluid collection were treated as control groups. Placental pathology was examined by HE staining, HMGB1 expression levels in placental tissue were measured by immunohistochemistry, PON-1 content in cord blood 
was measured by ELISA, and MCP-1 content in cord blood was determined by flow cytometry.

Specimen collection has been discussed by the Ethics Committee of Affiliated Hospital, Yanbian University to collect placenta and cord blood stained with term amniotic fluid stool delivered from January 2018 to December 2019. The total sample size was comprised of 73 subjects, 38 women in the MSAF group, and 35 women with term amniotic fluid serum in the control group. After specimen collection, all the placentae were stained with HE.

Placental collection method and storage: after the delivery of the maternal placenta, the placenta was extracted and fetal membrane tissue of about $3 \mathrm{~cm} \times 4 \mathrm{~cm}$ was collected. A $4 \%$ formaldehyde solution was taken for sealing and fixation. Paraffin and wax blocks were sectioned, 4 pieces with each cut thickness of 4-6 $\mu \mathrm{m}$, then sealed, and dried.

Process of umbilical cord blood collection: after delivery of the newborn, before breaking the umbilical cord from the mother, the umbilical cord was cut, and $5 \mathrm{ml}$ of the blood from the lateral umbilical vein was collected with a procoagulation tube. After centrifugation and centrifugation, the upper serum was collected, recorded with specimen information and numbers, and stored in the- $80{ }^{\circ} \mathrm{C}$ refrigerator.

\subsection{Degree of MSAF and Diagnostic Criteria of CAM}

2.2.1. The Standard of MSAF. Clinically, the amniotic fluid dung dye can be divided into $3^{\circ}$, from light to heavy and further, I degree, II degree, and III degree. I degree: light green, thin quality, and no faecium residue. Degree II : dark green, yellow-green, and relatively thick quality. Degree III : brown, dark yellow, thick, small amount, and visible granular meconium [11]. All the MSAF groups were stained with measured feces in this experiment.

2.2.2. The Diagnostic Criteria for CA. CA is further divided into acute and chronic chorioamnionitis. Acute chorioamnionitis could have neutrophils in the villus connective tissue, amniotic membrane, or chorionic plates. In chronic chorioamnionitis, lymphocytes infiltrate in the chorionic trophoblast and chorionic amniotic connective tissue [12].

2.2.3. Main Instrument, Equipment, and Reagents. The main instrument, equipment, and reagents used in the study have been mentioned in Table 1 .

2.2.4. Reagent. Cell Analysis Kit. High-throughput liquidphase protein quantification reagent of human CXCL8/IL-8, CCL5/RANTESCXCL9/MIQ, CCL2/MCP-1, CXCL10/IP1 cells: Becton, Dickinson, and Company.

Epidemiological histochemical experimental reagents:

(1) Rabbit anti-human HMGB1 clonal antibody: Cell Signaling Technology reagent agent

(2) DAB Kit: Abcam Reagent, Inc.
(3) PV9000 Kit: Abcam Kit Company, containing reagent 1: endogenous peroxidase blocker; reagent 2: reaction enhancer; Kit 3: HRP-enhanced goat antimouse/rabbit IgG polymer

ELISA Experimental Reagent. PON1 Quantitative ELISA Kit:Shanghai Enzyme Union Biotechnology Co., Ltd. The ELISA experimental reagents have been depicted in Table 2.

\subsection{Experimental Methods}

\subsubsection{Detection Steps of the Flow Cell Assay}

(1) Clean the flow cytometry to ensure the normal function of each instrument detection channel.

(2) Prepare standard solution and dilution: take $4 \mathrm{ml}$ standard dilution and freeze ophil dried to mix with the test tube, which is the original liquid, and rest for $10 \mathrm{~min}$. Nine standard tubes were set and one was blank, and the standards were subjected to proportional fold dilutions and added to 10 tubes and labeled S1-S 10. First take 300 ul from the raw liquid into the $\mathrm{S} 1$ tube, mix; then move from the $\mathrm{S} 1$ tube to $300 \mathrm{ul}$, and so on, until you move to the S10 tube. The dilution ratio and corresponding concentrations are shown in Table 3.

(3) Prepare capture microsphere suspension: IP-10 microsphere suspension was added for $1010 \mathrm{ul}$ to MCB tube, the supernatant was centrifuged for $5 \mathrm{~min}$, and serum was collected. Resuspension and incubation were done in light at room temperature for $20 \mathrm{~min}$.

(4) Take serum which has to be measured and further added to the tube for the test; microsphere suspension was added to standard products and serum samples to be measured and incubated for $3 \mathrm{~h}$ at room temperature

(5) Washing: add washing buffer for $1 \mathrm{ml}$ to each tube, and the supernatant was discarded. A 300 ul resuspension microball was added to each tube.

(6) The final standards and samples are analyzed for statistical data.

\subsubsection{ELISA Method}

(1) Sample serum: $-80{ }^{\circ} \mathrm{C}$, dissolved at room temperature, with shaking.

(2) Standard product and number: take standard product reagent, prepare 10 standard holes, and mark S1-S10. The same concentration was added to each two adjacent pore droplets such as S1, S2, and $50 \mu \mathrm{l}$, with concentrations of $120,80,40,20$, and $10 \mathrm{nmol} / \mathrm{mL}$, respectively.

(3) Set blank holes and additional samples: set as B1 and B2, except for blank holes; add $50 \mu$ sample to each sample hole to be tested.

(4) HRP: add $50 \mu \mathrm{l}$ of labeling reagent to each well. 
TABLE 1: Instrument name and manufacturer.

\begin{tabular}{|c|c|c|}
\hline & Instrument name & Manufacturer \\
\hline 1 & Leica automatic slicer & Leica, Germany \\
\hline 2 & PHY-III pathological tissue bleaching oven & Shanghai Changchang Electronics Co., Ltd. \\
\hline 3 & Electrothermal constant temperature incubator & Shanghai Yuejin photochemical instrument Factory \\
\hline 4 & Digital imaging equipment & Digital imaging equipment \\
\hline 5 & Micro oscillator & Xishan Jincheng Instrument Factory \\
\hline 6 & Foshan Zhiguang instrument Factory & Foshan Zhiguang Instrument Factory \\
\hline 7 & Ultrapure water machine & Shanghai Yuejin photochemical instrument Factory \\
\hline 8 & $-80^{\circ} \mathrm{C}$ refrigerator & Zhongke Meiling company \\
\hline 9 & Table type low speed refrigerated multitube centrifuge & Shanghai Anting Scientific Instrument Factory \\
\hline 10 & Micropipette & Mettler Toledo Instruments Co., Ltd. \\
\hline 11 & BioTek reader & Biotec instruments \\
\hline 12 & Incubator & Shanghai Xinmiao Medical Instrument Co., Ltd. \\
\hline 13 & Eppendorf micro sampler Eppendorf & Germany \\
\hline 14 & Flow cytometry & Beckton, Dickinson \\
\hline
\end{tabular}

TABle 2: PON1 quantitative ELISA kit components.

\begin{tabular}{lc}
\hline Intracellular kit reagent & \\
\hline 1 Instructions & Sample dilution: 1 bottle $(6 \mathrm{ml})$ \\
Seal plate film is 2 sheets & Color A and B 1 bottle each $(6 \mathrm{ml})$ \\
The plate was coated with 96 -well plates & Termination solution: 1 bottle $(6 \mathrm{ml})$ \\
Standard product (concentration of $120,80,40,20$, and $10 \mathrm{nmol} / \mathrm{mL}$, respectively) 5 bottles & Concentrate the detergent for 1 bottle \\
$(2 \mathrm{ml})$ & $(25 \mathrm{ml})$ \\
Enzyme labeling reagent 1 bottle $(6 \mathrm{ml})$ & \\
\hline
\end{tabular}

TABLE 3: List of corresponding concentrations and dilution ratio of standard tubes.

\begin{tabular}{lcc}
\hline Standard tube number & Corresponding concentration $(\mathrm{pg} / \mathrm{ml})$ & Dilution fold ratio \\
\hline S1 (blank control) & 0 & 0 \\
S2 & 10 & $1: 256$ \\
S3 & 20 & $1: 128$ \\
S4 & 40 & $1: 64$ \\
S5 & 80 & $1: 32$ \\
S6 & 156 & $1: 16$ \\
S7 & 312.5 & $1: 8$ \\
S8 & 625 & $1: 4$ \\
S9 & 1250 & $1: 2$ \\
S10 & 2500 & $1: 1$ \\
\hline
\end{tabular}

(5) Incubation: after gently shaking, the plate membrane was sealed and incubated in a $37^{\circ} \mathrm{C}$ incubator for 1 hour.

(6) Flushing: get rid of the liquid in the hole, fill with washing liquid, repeatedly wash and pat dry, and repeat 5 times in this way.

(7) Color display with the color developer: add color developer $\mathrm{A}, \mathrm{B} 50 \mu \mathrm{l}, 37^{\circ} \mathrm{C}$ incubators in each well to avoid light for 10 minutes.

(8) Color termination: gently for $50 \mu \mathrm{l}$, in each well.

(9) OD value of each well by computer.

2.4. The HMGB1 Immunohistochemical Staining Results Were Determined. HE staining mainly observed the morphology and pathological changes of the placenta and fetal membrane. In the placenta, HMGB1 is mainly localized in placental trophoblast, vascular endothelial cells as well as amniotic epithelial and stromal cells. The nucleus is positive in blue, and the cytoplasm is brown; negative if the nucleus, cytoplasm, or membrane is shown in blue[13]. Color intensity: The positive intensity of positive cells in each slice was 0 colorless, 1 pale yellow, 2 brown, and 3 dark brown. The average of positive cells: the percentage of positive cells was 0 , positive cell $<25 \% 1,25 \%-50 \% 2$, $>50 \%$ was 3 ; the sum of positive cells and color intensity: $0-1 ; 2-3(+) ; 4-5(++)$, and $6(+++)$.

2.5. Statistical Methods. All of the data were analyzed using SPSS 26.0 statistical software. Study data were analyzed using an independent sample $t$-test, grade data were analyzed using the nonparametric test, and $P<0.05$ was statistically significant. Risk factors analysis was performed using logistic regression analysis. 


\section{Results}

3.1. Comparison of Clinical Data between the MSAF and Control Groups. The total sample size comprised of 73 subjects. In the MSAF group, there were a total of 38 subjects, 22 men, 16 females, with the mean gestational age being $41.38 \pm 1.40$ weeks, and the control group was comprised of a total of 33 subjects, 19 men, 16 females, with the mean gestational age being $39.20 \pm 1.24$ weeks. The gender, birth weight, maternal age, initial/transmaternal, and gestational history were nonsignificant between both the groups $(P>0.05)$. However, gestational age, gestational diabetes, gestational hypertension, umbilical cord abnormalities, placental abnormalities, and neonatal asphyxia were significant between the groups $(P<0.05)$, shown in Table 4 .

Logistic regression analysis of the risk factor analysis in the MSAF group and gestational age and placental abnormalities were high-risk factors for amniotic fluid fecal staining, with OR being 2.639 (95\%CI:1.646-4.231) and 4.506 (95\%CI:1.034-19.631), respectively, and positively related (Table 5).

\subsection{Comparison of HMGB1 Expression and PON1, MCP-1,} and P. gingivalis Content between the MSAF Group and the Controls

3.2.1. HMGB1 Expression in the Placental Tissues of Both the MSAF and Control Groups. HMGB1 is mostly expressed in placental trophoblast, vascular endothelial cells, and amniotic epithelial and interstitial cells. The expression intensity of HMGB1 in the MSAF group was higher than in control tissues and was statistically significant $(P<0.05)$ as shown in Figures 1 and 2 and Table 6.

\subsubsection{Comparison of Umbilical Cord Blood PON1 Content in} the MSAF and Control Groups. Cord blood PON1 content in the MSAF group was $80.40 \pm 24.67 \mathrm{nmol} / \mathrm{mL}$ and in the control group was statistically significant $(P<0.001)$ as shown in Table 7.

3.2.3. Comparison of MCP-1 Plots and Content of Cord Blood in MSAF and Control Groups. Cord blood MCP-1 content was $271.10(174.35-326.62) \mathrm{pg} / \mathrm{mL}$, and the control was $104.89(50.15-184.19) \mathrm{pg} / \mathrm{mL}$, which was statistically significant $(P<0.001)$, shown in Figures 3 and 4 and Table 8.

3.3. Results of Placental HE Staining in Both the MSAF and Control Groups. After HE staining of 72 placentas, six had acute chorionic amnionitis (5 in MSAF, 1 in control), 32 had chronic chorioamnionitis (23 in MSAF, 9 in controls), 5 had nonabnormal placentas, and three in MSAF had chorionic amniotic columnar metaplasia (Figures 5-8).

\section{Discussion}

MSAF refers to the fact that when the fetal fetus is stimulated differently in utero, the intrauterine fetal intestinal peristalsis is enhanced, and when the anal sphincter is relaxed and the meconium is discharged into the amniotic fluid, this makes the amniotic fluid brown and yellow-green and then becomes cloudy and thick [14]. Fetal hypoxia causes intestinal peristalsis, the relaxation of the anal sphincter, and the release of meconium in the amniotic fluid. Meconium can also be sucked out in the first breath at birth, with studies suggesting that meconium induced direct alveolar damage through inflammatory responses and damaged lung parenchyma and endothelial cells and that $3 \%$ to $12 \%$ of infants born with MSAF would develop MAS, characterized by characteristic X-ray plaque shadows and respiratory distress and often exacerbated by pulmonary hypertension [15]. By collecting clinical data from pregnant mothers and newborns, this study found no difference between gender, birth weight, maternal age, maternal age, and maternal and gestational history, but gestational age, gestational diabetes, gestational hypertension, umbilical cord abnormalities, placental abnormalities, and neonatal asphyxia were statically significant. The gestational age and placental abnormalities were risk factors in the MSAF group and showed a positive correlation, indicating that the greater the gestational age, the more likely to develop MSAF. Lu Shaoxia et al.'s [1] study pointed out that simple amniotic fluid dung infection may not cause fetal distress. In 276 pregnant women with MSAF, the gestational week was found to be a high-risk factor for MSAF, with the incidence of pregnant women over 40 weeks, significantly greater than 38 weeks. Wang Li et al.'s [16] study showed that pregnant mothers had a higher incidence of gestational diabetes and preeclampsia in the MSAF group than in the normal group. MSAF can cause intrauterine oxygen and chronic hypoxia in the newborn and can cause different damage to the respiratory, circulation, and digestive systems of the nervous system of the newborn [17]. The above views are consistent with the present study. In the current study, umbilical cord abnormalities may also be the influencing factor of MSAF, spiral umbilical cord and knot, and tight winding, the umbilical cord is too short, and the incidence of placental inflammation and MSAF is significantly increased $[17,18]$. MSAF can be classified into primary and secondary contamination. After the fetal membrane rupture, amniotic fluid is feces, which is primary. After the fetal membrane rupture, the amniotic fluid is clear; along with the progress of the production process, the amniotic fluid gradually changes from brightening to feces dye, for secondary pollution [19]. Secondary meconium contamination cord was associated with meconium neonatal fetal distress and other poor neonatal prognoses, and primary amniotic fluid meconium contamination was associated with adverse outcomes [20]. Severe cases can die early in the newborn [21]. Microbial invasion of the amniotic cavity in placental inflammation increases the incidence of MSAF [22]. At present, the mechanism of amniotic fluid dung dyeing is unclear, and studying the correlation of inflammatory factors and MSAF can further explore the mechanism of MSAF occurrence.

The main diagnosis basis for diagnosing intrauterine infection is that the pathological testing of $\mathrm{CA}$ and the placenta can more effectively detect and judge intrauterine 
TABLE 4: Comparison of birth weight and maternal age of newborns in each group.

\begin{tabular}{|c|c|c|c|c|}
\hline Project & MSAF group (n, \%) & Control group (n, \%) & $\chi^{2}(t \%) Z$ & $P$ \\
\hline Fetal age $(x \pm s)$ & $41.38 \pm 1.40$ & $39.20 \pm 1.24$ & 7.053 & $<0.001$ \\
\hline Birth weight $(x \pm s)$ & $3.55 \pm 0.34$ & $3.41 \pm 0.40$ & 1.698 & 0.094 \\
\hline Mother age $(x \pm s)$ & $29.50 \pm 4.39$ & $30.00 \pm 5.11$ & 0.309 & 0.759 \\
\hline Gender (male/female) & $22 / 16$ & $19 / 16$ & 1.083 & 0.298 \\
\hline Early/via maternal & $13 / 25$ & $11 / 24$ & 0.064 & 0.804 \\
\hline Pregnancy diabetes & $12(31.6)$ & $4(11.4)$ & 4.323 & 0.038 \\
\hline Pregnancy hypertension & $15(39.5)$ & $5(14.3)$ & 5.811 & 0.016 \\
\hline Hyperthyroidism, hypothyroidism, and anemia & $6(15.8)$ & $5(14.3)$ & 0.032 & 0.858 \\
\hline Abnormal umbilical cord & $9(23.7)$ & $2(5.7)$ & 4.597 & 0.032 \\
\hline Abnormal placenta & $23(60.5)$ & $9(25.7)$ & 8.968 & 0.003 \\
\hline Neonatal asphyxia & $17(44.7)$ & $8(22.9)$ & 3.873 & 0.049 \\
\hline History of fetal protection & $9(23.7)$ & $7(20.0)$ & 0.145 & 0.704 \\
\hline
\end{tabular}

TABLE 5: Logistic regression analysis of the risk factor analysis in the MSAF group.

\begin{tabular}{lccccc}
\hline & $\mathrm{B}$ & SE & Wald & $P$ & OR (95\%CI) \\
\hline Fetal age & 0.971 & 0.241 & 16.247 & $<0.001$ & $2.639(1.646-4.231)$ \\
Pregnancy diabetes & 1.242 & 1.371 & 0.821 & 0.365 & $3.461(0.236-50.808)$ \\
Pregnancy hypertension & 0.753 & 1.114 & 0.457 & 0.499 & $2.122(0.239-18.823)$ \\
Abnormal umbilical cord & 1.139 & 1.217 & 0.876 & 0.349 & $3.123(0.287-33.932)$ \\
Abnormal placenta & 1.505 & 0.751 & 4.020 & 0.045 & $4.506(1.034-19.631)$ \\
Neonatal asphyxia & 1.295 & 0.782 & 2.742 & 0.098 & $3.653(0.788-16.926)$ \\
Constant & -40.592 & 9.827 & 17.062 & $<0.001$ & 0.000 \\
\hline
\end{tabular}

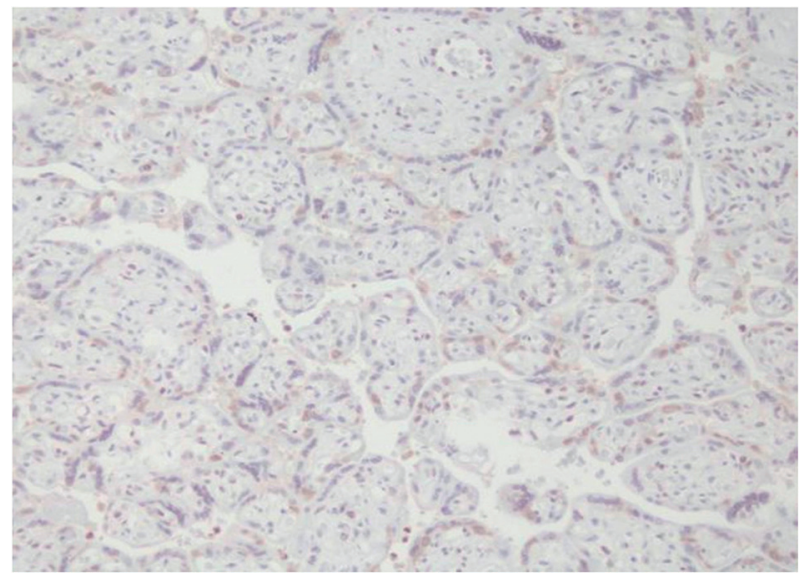

FIgURE 1: Expression of chorionic amniotic HMGB1 $(400 \times)$ in the control group.

infection [23, 24]. There was chronic inflammation of the placenta, compound trophoblast hyperplasia, and villus interstitial fibrosis in this experiment. There were CA, villus interstitial edema, fibrosis, and compound trophoblast hyperplasia; some studies pointed out that inflammatory cells with long span groups were mainly lymphocytes; that is, chronic inflammation is lymphocytes, and groups with short time span were neutrophils; namely, acute inflammation was mainly neutrophils [25]. In this study, it was shown that the MSAF group was more prone to placental inflammation, mainly with chronic inflammation.

HMGB1 is one member of the Alertin family, is divided in various cells, is an evolutionarily conserved DNA binding

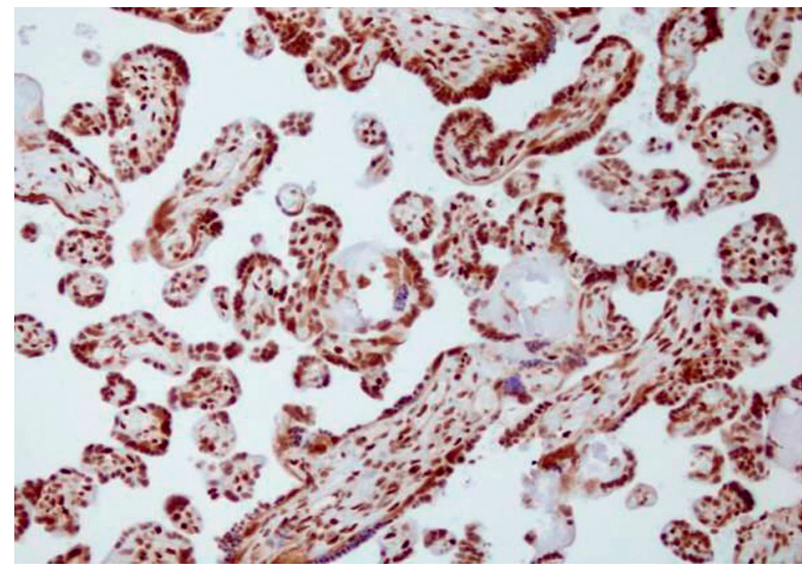

FIgURE 2: Expression of chorionic amniotic HMGB1 $(400 \times)$ in the MSAF group.

protein that acts as Alertin, is translocated to the cytoplasm, and is secreted after injury. Extracellularly, it acts as an inflammatory cytokine [26, 27]. In this study, HMGB1 expression in placentas is higher in the MSAF group than in the control by immunohistochemical staining of both placentas. Considering that HMGB1 has been implicated in placental inflammation, high HMGB 1 expression can upregulate the expression of late glycosylation end-product receptor (receptor for advanced glycation end products, RAGE), initiate a positive feedback mechanism, activate NF$\kappa \mathrm{B}$, to produce a series of inflammatory factors, and exacerbate the inflammatory response in the placenta [28]. HMGB1 may be involved in the occurrence of MSAF. 
TABLE 6: Comparison of HMGB1 expression intensity between the MSAF and control groups.

\begin{tabular}{|c|c|c|c|c|c|c|c|}
\hline \multirow{2}{*}{ Group } & \multirow{2}{*}{$N$} & \multicolumn{4}{|c|}{ HMGB1 expression intensity } & \multirow{2}{*}{$Z$} & \multirow{2}{*}{$P$} \\
\hline & & - & + & ++ & +++ & & \\
\hline MSAF group & 38 & $\begin{array}{c}1 \\
18\end{array}$ & $\begin{array}{c}7 \\
15\end{array}$ & 11 & 20 & 6.494 & $<0.001$ \\
\hline
\end{tabular}

TABle 7: Comparison of PON-1 content between the MSAF and control groups.

\begin{tabular}{lcc}
\hline Group & $\mathrm{N}$ & PON-1 $(\mathrm{nmol} / \mathrm{mL})$ \\
\hline MSAF group & 38 & $80.40 \pm 24.67$ \\
Control group & 35 & $95.65 \pm 24.33$ \\
$Z$ & & 2.658 \\
$P$ & & $<0.010$ \\
\hline
\end{tabular}

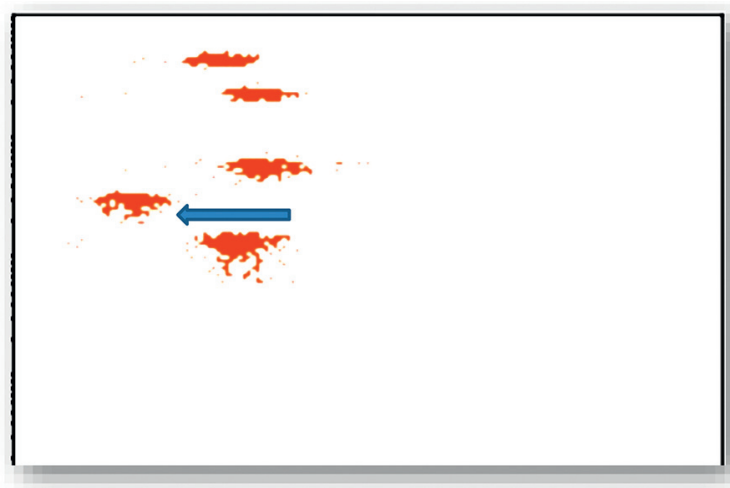

Figure 3: Cord blood MCP-1 content in the control group.

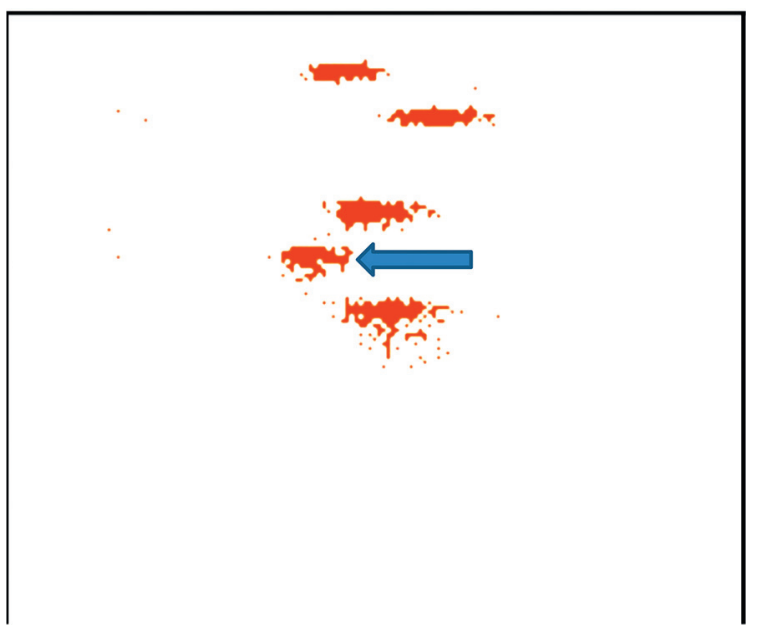

FIgURE 4: Cord blood MCP-1 content in MSAF group.

TABLE 8: Comparison of MCP-1 content between the MSAF and control groups.

\begin{tabular}{lcc}
\hline Group & $\mathrm{N}$ & $\mathrm{MCP}-1(\mathrm{pg} / \mathrm{mL})$ \\
\hline MSAF group & 38 & $271.10(174.35-326.62)$ \\
Control group & 35 & $104.89(50.15-184.19)$ \\
$\mathrm{Z}$ & & 4.03 \\
$\mathrm{P}$ & & $<0.001$ \\
\hline
\end{tabular}

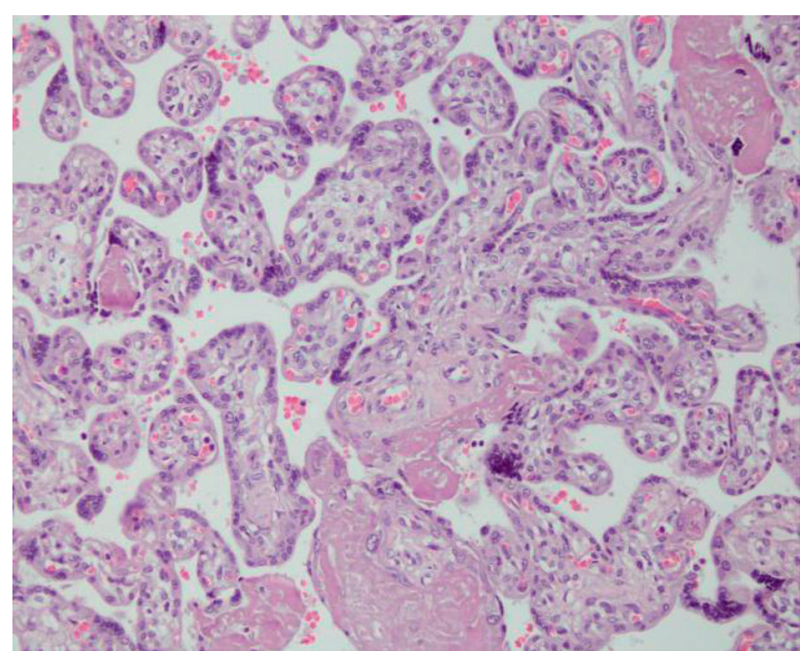

FIgURE 5: Chorioamnionitis $(200 \times)$.

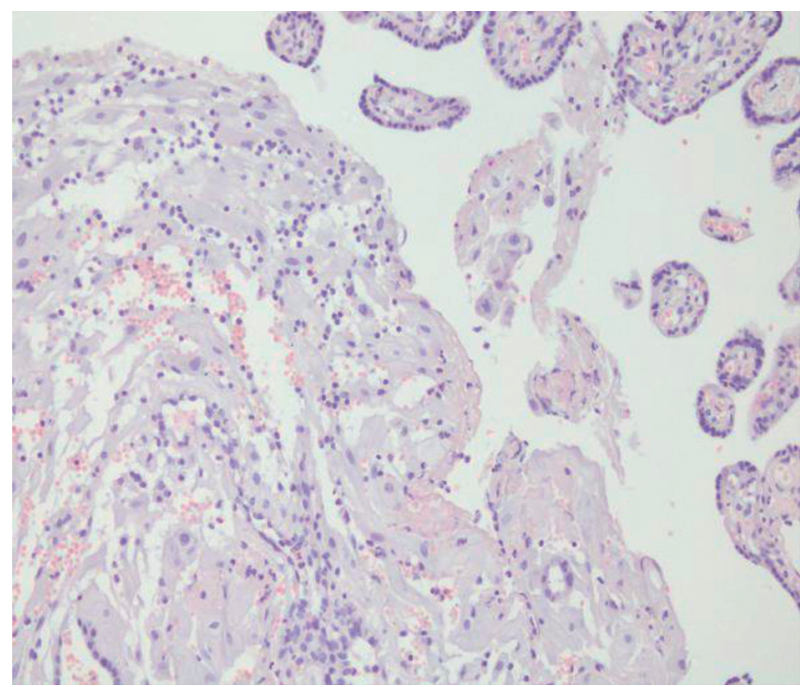

FIGURE 6: Chorioamnionitis with lymphocyte infiltration $(200 \times)$.

HMGB1 has been associated with pregnancy complications, such as preterm birth and CA [29, 30]. Salihu et al. [31] pointed out that HMGB1 can accelerate partial cellular senescence and enhance contractility in the myometry and expression of inflammatory genes. Endogenous hazard signals activate the Toll-like receptor-2 (TLR-2) and the Tolllike receptor-4 (TLR-4) and play a role in inflammatory diseases. HMGB1 may serve as an endogenous activator of these receptors. HMGB1 promotes neutrophils but not macrophage migration to necrotic tissue. In addition to the active secretion from inflammatory cells, HMGB1 is also passively released from necrotic cells, and HMGB1 from 


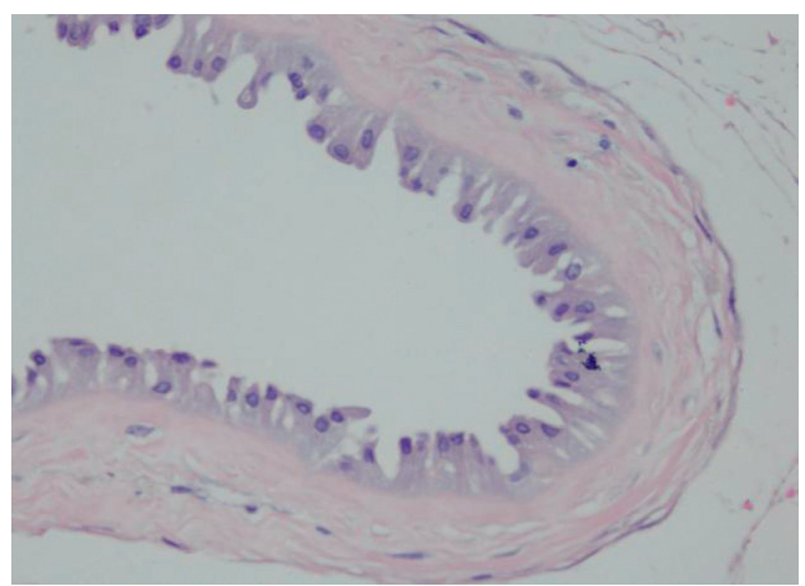

Figure 7: Chorioamniotic columnar epithelialization $(400 \times)$.

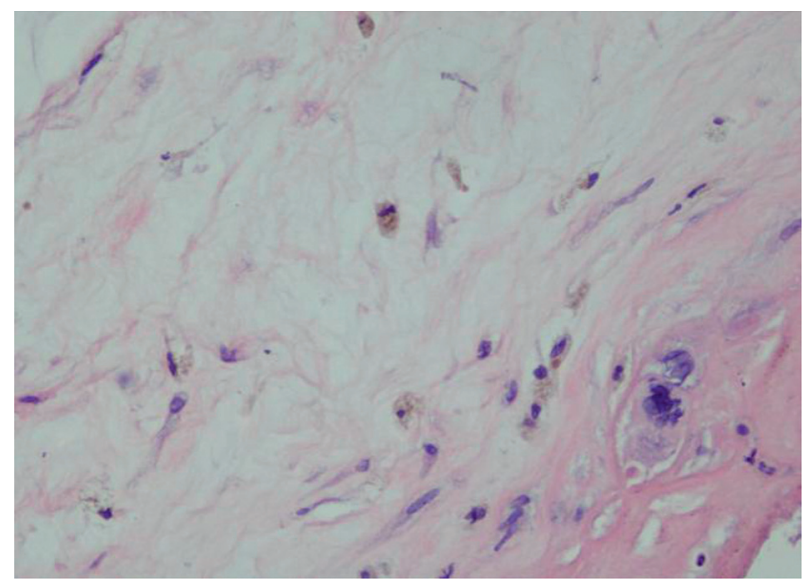

FIGURE 8: Placental chorioamnionitis edema in the MSAF group $(400 \times)$.

cells at different sites can affect the inflammatory response after necrosis to varying degrees [32]. HMGB1 can promote the release of inflammation, with redox and endogenous cytokines-induced functional extracellular TLR4 mainly expressed in placental compound trophoblasts and fibroblasts. HMGB1 induces macrophages to produce inflammatory cytokines in a redox-dependent manner through TLR4 signaling, affecting cell proliferation, differentiation, and migration [26,33]. All indicate a correlation with the occurrence of inflammation. HMGB1 can form an immunostimulatory complex. The inflammatory response triggered by HMGB1 may be involved in the placental inflammatory response that can induce the production of transcription factors (e.g., NF- $\kappa \mathrm{B}$ ) and trigger a local (and subsequently systemic) inflammatory response [34]. TLR2 and TLR4 are associated with MSAF, and in inflammatory cells, the Toll-like receptor (TLR) is combined with its ligand (HMGB1) through the recruitment of linker proteins to strongly activate NF-kB signaling, triggering inflammation [35]. The activity of HMGB1 that depends largely on its redox state, reduces HMGB1 release, and chemically modifies its redox form can improve inflammation as well as tissue damage. A positive correlation was observed between the expression level of reactive oxygen species and the expression of HMGB1 protein [36]. In the MSAF study, HMGB1 expression in the placenta was stronger than in the control group, reliable, and oxidized.

PON-1, a member of the antioxidant enzyme family with anti-inflammatory effects, is an effective antioxidant associated with the pathogenesis of multiple diseases [37]. Multiple pathophysiological diseases are related and also have the damage of degraded organophosphate compounds to the nervous system $[38,39]$. Thus, the state of PON1 in vivo can be determined by measuring the levels of enzymatic activity with different substrates [40,41]. In this study, the PON1 gene is closely related to poison metabolism, and the strength of its activity directly affects the poison metabolism process in vivo. The oxidative stress response is involved in the development of inflammation and is an important cause of the expansion and aggravation of inflammatory response. The interaction of oxidative stress with the inflammatory response is the mechanism of disease development. Oxidative products are produced in normal physiological metabolism, while cells express endogenous antioxidants to remove free radicals and combat the harmful effects of reactive oxygen species products, keeping the oxidation/antioxidant system in a stable, balanced state [42]. PON-1 is a marker in oxidative stress metabolites. In the state of oxidative stress, the measured PON-1 content of cord blood in this study showed lower PON1 activity in the MASF group than normal group, considering the oxidative/ antioxidant system imbalance and the occurrence of oxidative stress and inflammation. Shandan et al. [43] pointed out that oxidative stress can cause placenta ischemia and hypoxia and affect the release of inflammatory factors and related enzymes. The possible involvement in the oxidative stress response in causing inflammation may have some relevance to MSAF.

MCP-1 is chemotaxis immune cells that produce corresponding antibodies after stimulation and affect phagocytosis to resist the invasion of foreign microorganisms. In recent years, studies have pointed out labor initiation, preterm birth, and pregnancy-related diseases $[44,45] . \mathrm{Xu}$ Qingyun [46] suggests that elevated MCP-1 in neonatal cord blood may be involved in the development of CA. The main factor of CA is inflammatory factors through signaling; oxidative stress can stimulate inflammation and fetal edema degeneration; edema appeared in this study; there was placental HE staining; three cases had columnar metaplasia and MSAF cord blood MCP-1 higher than the control group and statistically significant difference; for placental $\mathrm{HE}$ staining, 3323 cases in the MSAF group had placental CA and mainly chronic chorioamnionitis. Case analysis studies studying preterm birth with MSAF indicated a higher probability of CA compared with the MSAF group [47, 48]. Liu Weiwei [14] pointed out that pregnant women in the MSAF group had a higher probability of neonatal infection than in the CA-free group; CA increased neonatal infection rate, increased intrauterine infection index in the perinatal period, and increased risk of infection during fetal delivery. Yan Lili et al. [49] pointed out that MCP-1 can drive activated macrophages and, with high MCP-1 expression, 
increases cell adhesion and invasion and aggravates inflammation. In pediatric bronchial asthma, oxidative stress status is correlated with it, among which MCP-1 increases significantly between the onset and remission period in children, pointing out that oxidative stress levels are correlated with the imbalance of inflammatory factors [50]. MCP-1 may have some correlation with MSAF [9].

4.1. Limitations. The small sample size is limited, and increasing the sample size can improve the relevant studies.

\section{Conclusion}

The study concluded that gestational age and placental abnormalities are clinical high-risk factors for MSAF. We have found that placental chorioamnionitis may be one of the causes of MSAF. HMGB1, PON-1, MCP-1, and $P$. gingivalis may be involved in the development of MSAF, suggesting an oxidative/antioxidant imbalance with inflammation, and may be one of the mechanisms of MSAF development.

5.1. Practical Implications. HMGB1 PON-1, MCP-1, and $P$. gingivalis play an important role in immune responses in MSAF patients [51].

\section{Data Availability}

The collected data have been included in the study.

\section{Ethical Approval}

Specimen collection has been discussed by the Ethics Committee of Affiliated Hospital, Yanbian University, to collect placenta and cord blood stained with term amniotic fluid stool delivered from January 2018 to December 2019.

\section{Conflicts of Interest}

The authors declare that they have no conflicts of interest.

\section{Acknowledgments}

This study was supported by the Biostime Nutrition and Nursing Home "Maternal and Infant Clinical Research Special Fund" under 2020BINCLC009 and Science and Technology Project of Yanbian University.

\section{References}

[1] S. Lu, S. Ou, and X. Kang, "Case of clinical analysis," Modern Chinese Drug Application, vol. 18, no. 21, pp. 264-265, 2015.

[2] J. Xu, "The relationship between perinatal high-risk factors and fetal distress and neonatal asphyxia," Chinese Practical medicine, vol. 10, no. 24, pp. 276-277, 2015.

[3] S. Yue, "Amniotic fluid meconium pollution and nervous system damage," Chinese Clinical Journal of Practical Pediatrics, vol. 30, no. 14, pp. 1046-1050, 2015.
[4] L. Yin, "Study and analysis of perinatal high risk factors and fetal intrauterine distress and neonatal asphyxia," Journal of Aerospace Medicine, vol. 28, no. 4, pp. 404-405, 2017.

[5] $\mathrm{M}$. Wu, "Amniotic fluid meconium pollution and neonatal prognosis," Women and abroad, vol. 11, no. 18, pp. 36-37, 2016.

[6] J. Lee, R. Romero, K. A. Lee et al., "Meconium aspiration syndrome: a role for fetal systemic inflammation," American Journal of Obstetrics and Gynecology, vol. 214, no. 3, pp. e1366, 2016.

[7] L. Chen, C. Geng, and L. Zhang, "The relationship between contamination and duration of meconium and placental pathological changes," Clinical Medical Engineering, vol. 22, no. 10, pp. 1367-1368, 2015.

[8] M. Huang, M. Guo, K. Wang et al., "HMGB1 mediates paraquat-induced neuroinflammatory responses via activating RAGE signaling pathway," Neurotoxicity Research, vol. 37, no. 4, pp. 913-925, 2019.

[9] X. Qiu, Significance of HMGB1 and its receptor in Chorioamnionitis-Related Preterm Birth, Tianjin Medical University, Tianjin, China, 2017.

[10] M. Mutlu, M. H. Korkmaz, E. Simsek et al., "Do CO2 and oxidative stress induce cancer?: a brief study about the evaluation of PON 1, CAT, CA and XO enzyme levels on head and neck cancer patients," Journal of Enzyme Inhibition and Medicinal Chemistry, vol. 34, no. 1, pp. 459-464, 2019.

[11] Y. Wu and Z. Wang, "Clinical significance and treatment of amniotic meconium pollution," Chinese Journal of Perinatal Medicine, vol. 15, no. 4, pp. 203-205, 2012.

[12] C. J. Kim, R. Romero, P. Chaemsaithong, and J.-S. Kim, "Chronic inflammation of the placenta: definition, classification, pathogenesis, and clinical significance," American Journal of Obstetrics and Gynecology, vol. 213, no. 4, pp. S53-S69, 2015.

[13] S. Ni and J. Liu, "Expression of matrix metalloproteinases and tissue inhibitors and Toll-like receptors in fetal membrane tissue in patients with premature fetal membrane rupture," Hebei Medicine, vol. 40, no. 1, pp. 28-31, 2018.

[14] W. Liu, W. Zhang, and Y. Jiao, "Effect of amniotic fluid meconium contamination and chorionic amnionitis on maternal and infant infection," Chinese Journal of Neonatology (Chinese and English), vol. 34, no. 4, pp. 281-285, 2019.

[15] G. Lista, ““”NNeonatologists and non-vigorous newborns with meconium-stained amniotic fluid (MSAF) in the delivery room: time for hands off?" European Journal of Pediatrics, vol. 178, no. 12, pp. 1823-1824, 2019.

[16] L. Wang, "Case of meconium contamination," Medical Information, vol. 29, no. 33, pp. 274-275, 2016.

[17] H. Chen and S. Chen, "Discuss the influence of amniotic fluid manure infection on maternal pregnancy outcome and correlation," Modern drug application in China, vol. 13, no. 12, pp. 23-25, 2019.

[18] Y. Zhang, "Amniotic meconal contamination and fetal intrauterine distress and neonatal asphyxia," Health Care Guide, vol. 10, no. 40, p. 363, 2018.

[19] L. Hiersch, N. Melamed, H. Rosen, Y. Peled, A. Wiznitzer, and Y. Yogev, "New onset of meconium during labor versus primary meconium-stained amniotic fluid - is there a difference in pregnancy outcome?" The Journal of Maternal-Fetal \& Neonatal Medicine, vol. 27, no. 13, pp. 1361-1367, 2014.

[20] Q. Xu, "Clinical significance of primary and secondary amniotic fluid meconium contamination," Medical Theory and Practice, vol. 31, no. 6, pp. 875-877, 2018. 
[21] M. Ali, A. Maruf, N. Naher, and S. Islam, "Neonatal outcome in meconium stained amniotic fluid (MSAF): a study in a neonatal high dependency unit (NHDU) of a medical college hospital," Mediscope, vol. 6, no. 2, pp. 65-71, 2019.

[22] D. Paz-Levy, A. Walfisch, T. Wainstock, D. Landau, and E. Sheiner, "'696: meconium stained amniotic fluid exposure is associated with a lower rate of offspring's infectious morbidity," American Journal of Obstetrics and Gynecology, vol. 220, no. 1, pp. S459-S460, 2019.

[23] M. Bolten and E. Chandraharan, "The significance of 'nonsignificant' meconium stained amniotic fluid (MSAF): colour versus contents," Journal of Advances in Medicine and Medical Research, vol. 30, no. 5, pp. 1-7, 2019.

[24] H. Liao, "Analysis of placental disease infected with amniotic fluid dung," Everybody Health (Academic Edition), vol. 10, no. 7, pp. 58-59, 2016

[25] J. Zheng, L. Zheng, D. Wang et al., "The relationship between amniotic fluid dung dyeing and amniotic cavity infection," Chinese Journal of Infectology of the Hospital, vol. 23, no. 8, pp. 1846-1847, 2013.

[26] A. Raucci, S. Di Maggio, F. Scavello, A. D’Ambrosio, M. E. Bianchi, and M. C. Capogrossi, "The Janus face of HMGB1 in heart disease: a necessary update," Cellular and Molecular Life Sciences, vol. 76, no. 2, pp. 211-229, 2019.

[27] H. Yang, P. Lundbäck, L. Ottosson et al., "Expression of Concern to: redox modification of cysteine residues regulates the cytokine activity of high mobility group box-1 (HMGB1)," Molecular Medicine, vol. 26, no. 1, p. 18, 2020.

[28] L. Y. Huang, I. C. Yen, W. C. Tsai et al., "Rhodiola crenulata suppresses high glucose-induced matrix metalloproteinase expression and inflammatory responses by inhibiting ROSrelated HMGB1-TLR4 signaling in endothelial cells," The American Journal of Chinese Medicine, vol. 48, pp. 1-15, 2020.

[29] R. Menon, F. Behnia, J. Polettini, G. R. Saade, J. Campisi, and M. Velarde, "Placental membrane aging and HMGB1 signaling associated with human parturition," Aging, vol. 8, no. 2, pp. 216-230, 2016.

[30] C. Hernandez, P. Huebener, J. P. Pradere et al., "HMGB1 links chronic liver injury to progenitor responses and hepatocarcinogenesis," The Journal of clinical investigation, vol. 128, no. 6, pp. 2436-2450, 2019.

[31] H. M. Salihu, A. Pradhan, L. King et al., "Impact of intrauterine tobacco exposure on fetal telomere length," American Journal of Obstetrics and Gynecology, vol. 212, pp. 205-208, 2015.

[32] Y. Wang, F. Du, A. Hawez et al., "Neutrophil extracellular trap -microparticle complexes trigger neutrophil recruitment via high -mobility group protein 1 (HMGB1)-oll -like receptors (TLR2)/TLR4 signalling," British Journal of Pharmacology, vol. 176, no. 17, pp. 3350-3363, 2019.

[33] B. Khambu, N. Huda, X. Chen et al., "HMGB1 promotes ductular reaction and tumorigenesis in autophagy-deficient livers," The Journal of clinical investigation, vol. 128, no. 6, pp. 2419-2435, 2019.

[34] S. Rakoff-Nahoum and R. Medzhitov, "Toll-like receptors and cancer," Nature Reviews Cancer, vol. 9, no. 1, pp. 57-63, 2009.

[35] S. Zhang, M. Wang, Z. Chen et al., "Expression of Toll-like receptor in children with hemophocytic syndrome," Chinese Journal of Experimental Hematology, vol. 27, no. 5, pp. 1664-1671, 2019.

[36] L. Yan, S. Z. Qi, and T. Wang, "Regulation of HMGB1 expression in lung cancer cells and its application value in lung cancer screening," Journal of Clinical Examination, vol. 37, no. 8, pp. 625-628, 2019.
[37] M. Alimohammadi, M. Soodi, and M. Gholami Fesharaki, "Organophosphate pesticide exposure reduced serum Paraoxonase1 (PON1) activity which correlated with oxidative stress in pesticide factory workers," Archives of Hygiene Sciences, vol. 8, no. 2, pp. 88-97, 2019.

[38] S. Dong, Z. Liu, and S. Guo, "Effect of PON1 activity and AOPP levels on disease severity in preeclampsia," Maternal and Child Health Care in China, vol. 34, no. 12, pp. 2698-2701, 2019.

[39] Y. Wu, "Study on diagnosis of PON1 diagnosis and disease in women with endometriosis," Experimental and Laboratory Medicine, vol. 37, no. 5, pp. 919-921, 2019.

[40] X. Xiao, X. Wen, Y. Chu et al., "PON-1 reduces the molecular mechanism of myocardial oxidative stress damage by upregulating HSPB1 expression," Journal of Practical Shock (Chinese and English), vol. 3, no. 3, pp. 150-154, 2019.

[41] C. O. Reichert, C. G. de Macedo, D. Levy et al., "Paraoxonases (PON) 1, 2, and 3 polymorphisms and PON-1 activities in patients with sickle cell disease," Antioxidants, vol. 8, no. 8, p. 252, 2019.

[42] G. Lin and H. Zhao, "Effect of oxidative stress on placental life," International Journal of Obstetrics and Gynecology, vol. 46, no. 2, pp. 185-188, 2019.

[43] D. Shi, Y. Wang, J. Guo et al., "Clinical study on oxidative stress, inflammatory response, and vascular endothelial injury in patients with preeclampsia," PLA Journal of Medicine, vol. 30, no. 1, pp. 60-63, 2018.

[44] S. Lin, D. Ke, Y. Lin et al., "Puerarin inhibits the migration of osteoclast precursors and osteoclastogenesis by inhibiting MCP-1 production," Bioscience, Biotechnology, and Biochemistry, vol. 84, pp. 1-5, 2020.

[45] C. Sun, X. Li, E. Guo et al., "MCP-1/CCR-2 axis in adipocytes and cancer cell respectively facilitates ovarian cancer peritoneal metastasis," Oncogene, vol. 39, no. 8, pp. 1681-1695, 2020.

[46] Q. Xu, Correlation between TLR-2, 4 and MCP-1 and Premature Fetal Membrane Rupture and Chorioamnionitis, Yanbian University, Jilin, China, 2018.

[47] D. Brabbing-Goldstein, D. Nir, D. Cohen, A. Many, and S. Maslovitz, "Preterm meconium-stained amniotic fluid is an ominous sign for the development of chorioamnionitis and for in utero cord compression," The Journal of Maternal-Fetal \& Neonatal Medicine, vol. 30, no. 17, pp. 2042-2045, 2017.

[48] K. Abraham, E. Thomas, and J. Lionel, "New evidence to support antibiotic prophylaxis in meconium-stained amniotic fluid in low-risk women in labor a prospective cohort study," The Journal of Obstetrics and Gynecology of India, vol. 68, no. 5, pp. 360-365, 2018.

[49] L. Yan and H. Lijuan, "Changes and significance of peripheral blood MCP-1, sflt-1, sICAM-1 in patients with endometriosis," Chinese Journal of Family Planning, vol. 27, no. 10, pp. 1339-1342, 2019.

[50] C. Zhu and L. Zhang, "Evaluation of serum inflammatory factor levels and oxidative stress status in children with bronchial asthma," World Composite Medicine, vol. 5, no. 8, pp. 33-35, 2019. 